\title{
Entorno y motivaciones para emprender
}

FECHA DE RECEPCIÓN: 27 de julio

FECHA DE APROBACIÓN: 26 de agosto pp. $12-28$

DOI: http://dx.doi.org/10.21158/01208160.n81.2016.1556

\section{Resumen}

Este estudio está orientado a profundizar en el análisis de las motivaciones en un grupo de emprendedores de Medellín (Antioquia-Colombia), así como en la percepción sobre facilitadores y obstáculos para el emprendimiento en un entorno en el que se han implementado diversos mecanismos para su fomento. Se encontró que la independencia fue la principal motivación y que se considera que la ciudad ofrece un ambiente favorable para esta actividad. A su vez, el mayor obstáculo que se percibe es la dificultad para acceder al crédito bancario.

\section{Palabras clave}

Emprendimiento, motivaciones, entorno, facilitadores, obstáculos.

\section{Flor Ángela Marulanda Valencia}

Doctorado en Ingeniería Industrial y Organizaciones, Universidad Nacional de Colombia; Maestría en Ciencias de la Administración, Universidad EAFIT; Especialización en Gestión de la Calidad Universitaria, Universidad de San Buenaventura; Especialización en Finanzas, Preparación y Evaluación de Proyectos, de la Universidad de Antioquia; Ingeniera Administrativa, Universidad Nacional de Colombia.

\section{Silvia Teresa Morales Gualdrón}

Ph.D. en Dirección de Empresas; profesora asociada grupo de investigación en Finanzas, Emprendimiento y Gestión Organizacional, del Departamento de Ingeniería Industrial, Facultad de Ingeniería, Universidad de Antioquia. 


\section{Conditions and Motivations to Undertake}

Abstrac. This study aims at deepening in the analysis of motivations shown by a group of entrepreneurs in Medellin, Antioquia. It also describes the different perceptions about the enablers and obstacles for the development of entrepreneurship in appropriate environments to promote it. It was found that independence was the principal motivation for entrepreneurship and that the city offered the most favourable environment to foster it. Additionally, it was found that the most important obstacle to develop it was the difficulties to access a bank credit.

Key words. Entrepreneurship, motivations, environment, enablers, obstacles.

\section{Environnement et motivations propices à l'entrepreneuriat}

Resumé. Cette étude a pour but d'approfondir l'analyse des motivations d'un groupe d'entrepreneurs de Medellín (Antioquia - Colombie), leur perception des facteurs facilitateurs et des obstacles à l'entrepreneuriat dans un environnement où différents mécanismes ont été mis en place pour accroitre le succès de ces expériences. L'indépendance professionnelle semble être la motivation principale de ce groupe dans une ville offrant un climat favorable à l'activité entrepreneuriale. Le principal obstacle perçu reste la difficulté d'accès au crédit bancaire.

Mots clefs. Entrepreneuriat, motivations, environnement, facilitateurs, obstacles.

\section{Ambiente e motivações para empreender}

Resumo. O seguinte estudo está orientado ao aprofundamento da análise das motivações em um grupo de empreendedores de Medellín (Antioquia, Colômbia), mas também à percepção sobre os facilitadores e os obstáculos para o empreendimento, em um ambiente no qual se implementaram diversos mecanismos para seu estímulo. Encontrou-se que a diferença que a principal motivação era a independência, e ainda consideram que a cidade oferece um ambiente favorável para esta atividade. Por sua vez, o maior obstáculo que percebem é a dificuldade para obter o crédito bancário.

Palabras chave. Empreendimento, motivações, ambiente, facilitadores, obstáculos. 


\section{Introducción}

\section{b}

a generación de nuevas empresas se ha $\checkmark$ convertido en un reto permanente para las diferentes instancias gubernamentales, por cuanto existe una necesidad de generar fuentes de empleo y riqueza de forma permanente, que coadyuven al mejoramiento de las condiciones socioeconómicas de las comunidades, en el entendido de que el emprendimiento es un motor de desarrollo. En este contexto, los emprendedores se convierten en un eje fundamental, puesto que son ellos los que identifican las oportunidades y actúan en pro de su aprovechamiento. Así pues, el fomento de la actividad emprendedora requiere del estudio de motivaciones y percepciones propios del contexto local, ya que, además de los factores universales que se describen en la literatura, existen otros más específicos de cada país relacionados con los aspectos económicos, culturales, educativos, así como las condiciones de fomento al emprendimiento y las capacidades emprendedoras en la población (Kantis, Angelelli \& Gatto, 2001).

En el estudio de las motivaciones para emprender, se han utilizado diferentes clasificaciones; una de las más reconocidas es la que se hace de acuerdo con la fuente de ellas, según la cual pueden ser de tipo intrínseco o extrínseco. Las de tipo extrínseco son aquellas que vienen desde fuera del individuo. En este grupo se incluye la existencia en el entorno de facilitadores para la creación de empresas. En el caso colombiano, durante los últimos años, se ha visto un fortalecimiento de las capacidades para el fomento de la cultura emprendedora y la creación de empresas. De hecho, en el año 2006, se promulgó la Ley 1014 que creó un marco interinstitucional para el fomento del emprendimiento y la creación de empresas.
Concretamente, en Medellín, se han creado programas y entidades, muchas de ellas englobadas dentro del programa Cultura E, cuyo objetivo es promover la cultura emprendedora a través de servicios de sensibilización, formación, acompañamiento, financiación y acceso a mercados. Considerando estos esfueros, es de especial interés estudiar las motivaciones para emprender y la incidencia del entorno desde la perspectiva del emprendedor/ empresario en dichas motivaciones. Igualmente de relevante, es el conocer las percepciones de los emprendedores con relación a las facilidades que dichas instituciones y programas ofrecen para la creación de empresas, así como los obstáculos existentes, pues como afirman Capelleras et al. (2013), "las percepciones subjetivas de los emprendedores han servido para explicar la decisión al emprender una nueva iniciativa empresarial».

Con el fin de avanzar en el análisis de las motivaciones para emprender y las percepciones del entorno institucional, este trabajo presenta los resultados de un estudio exploratorio realizado con 18 emprendedores adscritos al programa de emprendimiento de la Universidad Nacional, y pretende responder los siguientes cuestionamientos: ¿cuáles son las motivaciones que llevan a las personas a crear y gestionar sus empresas? y ¿cuál es la percepción que los

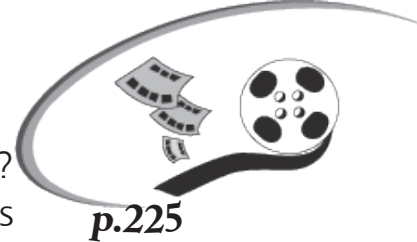
micro y pequeños empresarios tienen sobre el entorno que presenta la ciudad de Medellín, en cuanto a las facilidades y obstáculos para la actividad emprendedora? 


\section{La importancia de las motivaciones en el proceso emprendedor}

D esde el punto de vista teórico, uno de los planteamientos más aceptados sobre el emprendimiento es el propuesto por Paturel (1997), quien se basa en un esquema que denomina "La coherencia del proyecto». De acuerdo con este, para que se dé efectivamente la creación de una empresa deben confluir tres factores: las aspiraciones o motivaciones del creador; sus competencias -habilidades y Know-how-y recursos -financieros y logísticos, red de relaciones-; y el ambiente o entorno para la creación, que se refiere, específicamente, a las oportunidades que ofrece el mercado y los incentivos que brinda el marco legal para la actividad emprendedora (Figura 1). En el punto $A$ se presenta la confluencia de los tres factores; la ubicación del proyecto por fuera de esta área indicará que este no es viable. El punto $B$ indica que se tiene la motivación y los recursos para crear la empresa, pero el entorno no es favorable. En el punto $C$ se tienen los recursos y la favorabilidad del ambiente, pero el potencial creador no se siente suficientemente motivado por el proyecto como para desarrollarlo. En el punto $D$ están presentes la motivación del creador y el ambiente favorable, pero se carece de recursos para ejecutar el proyecto.

Figura 1. Esquema de la coherencia del proyecto con la zona de compatibilidad del creador.

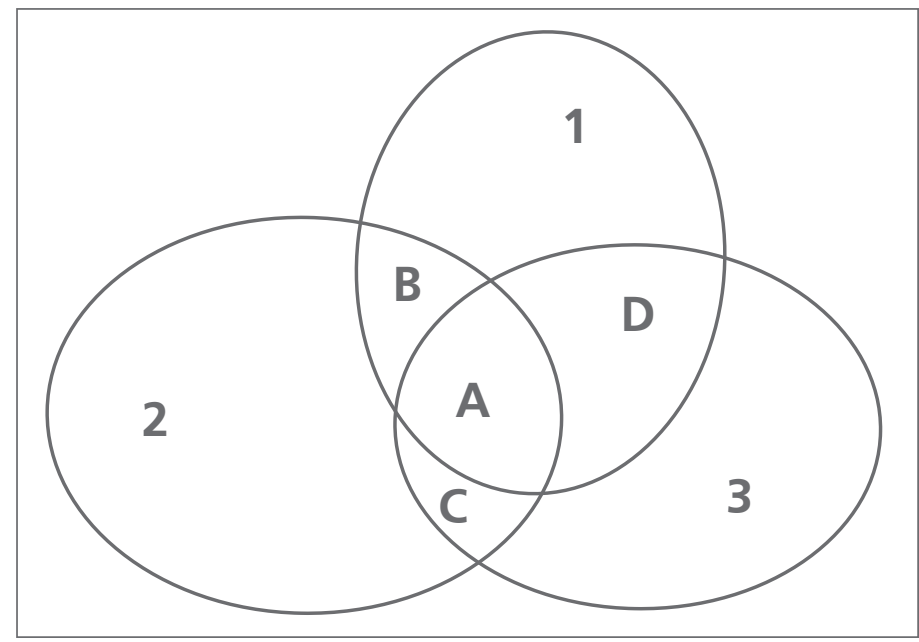

Fuente. Paturel (1997, p. 15).

Podría decirse que el factor más importante es la motivación, pues esta es la fuerza que impulsa a los emprendedores a vencer las dificultades relacionadas con los otros dos factores (Marulanda, 2015), y por esto, su estudio despierta gran interés en los académicos, lo que debe extenderse a las instituciones que promueven y dirigen los programas de apoyo a la creación de empresas (Orrego, 2008).

En el área del comportamiento humano, la motivación ha sido estudiada con el fin de 
responder a tres tipos de preguntas: lo que activa a una persona, lo que hace que el individuo elija una conducta sobre otra, y por qué diferentes personas responden de manera distinta a los mismos estímulos motivacionales (Carsrud \& Brännback, 2011). Palmero (2005), señala que la motivación ostenta aspectos biológicos y culturales, pues muchos de los desafíos adaptativos a los que se enfrenta el ser humano tienen connotaciones sociales. Así, las metas dirigen al individuo dependiendo de las condiciones del estímulo; la motivación por su parte, moviliza las acciones pertinentes.

Palmero (2005), hace referencia a tres componentes de la motivación, los cuales, en interacción mutua y con el medio ambiente, posibilitan la activación y dirección de la conducta motivada, a saber: a) el componente biológico fundamentado en el hecho de que la conducta, en parte, depende de la estructura genética del sujeto; b) el componente de aprendizaje o motivos adquiridos, como el logro y el poder; y c) el componente cognitivo relacionado con los procesos de conocimiento. De igual forma, la percepción, bien sea consciente o no consciente, es una variable imprescindible para que ocurra el proceso motivacional.

\subsection{Factores endógenos y factores exógenos como motivadores para el emprendimiento}

El psicólogo David McClelland (1961), quien fue uno de los primeros en analizar el tema de la motivación de los emprendedores desde una perspectiva más allá del aspecto puramente económico, afirma que la necesidad de logro es lo que realmente lleva a un individuo a convertirse en emprendedor. Según el autor, el desarrollo de esta atribución está influenciado en la persona por la crianza y por aspectos sociales y culturales del entorno. Su aporte consistió, además de descubrir que este rasgo de la personalidad es indispensable para ser un emprendedor de éxito, en demostrar que este no es necesariamente innato, sino que se puede desarrollar (McClelland, 1965).

En esta misma vía, Martínez, Mira y Gómez (2012) señalan, que si bien, el individuo es el factor clave en el proceso de creación de una empresa, por ser quien toma la decisión y desarrolla las actividades correspondientes, este proceso ocurre en un contexto dado, por lo cual, al analizar este tema es necesario tener en cuenta tanto los rasgos individuales del emprendedor como la influencia de las características del entorno.

Shapero (1985), cuya teoría del evento empresarial se ha convertido en una de las bases principales del estudio del emprendimiento, afirma que la decisión de crear empresa es el producto de dos percepciones: conveniencia o deseabilidad, y factibilidad o viabilidad. Las percepciones de conveniencia tienen que ver con los valores y son el resultado de la posición de la persona en la matriz de cultura, estructura socioeconómica, familia, educación, pares y personas influyentes. Afirma que son más propensas a la creación de empresas aquellas culturas que valoran el ser negociante, el emprendimiento y los comportamientos asociados a estas activi. dades, como la independencia, la innovación y la toma de riesgos. Esto puede suceder, no solo a nivel de países, sino que es posible referirse a subculturas, es decir, regiones dentro de aquellos, que forman sus propios sistemas de valores; como ejemplos señala, entre otras, los regiones de Smoeland en Suiza, Cork en Irlanda y Antioquia en Colombia (Shapero, 1985, p. 26). 
En cuanto a la percepción de factibilidad, esta es considerada por el autor como una mezcla de conocimientos, recursos, garantías y experiencia obtenida en un negocio particular o en la educación formal. Muchos emprendedores crean empresas en sectores en los cuales han laborado, así mismo, cuando se trabaja en empresas pequeñas se tiene un conocimiento más integral del negocio, y, por tanto, hay mayor inclinación a crear el propio. También, mejora la percepción de factibilidad el haber tenido contacto con clientes y un acercamiento al mercado. Pero para el autor la mayor influencia en la percepción de factibilidad proviene de la observación de otros: ver que otros similares a uno hacen ciertas cosas es más motivador que simplemente imaginarse a uno mismo haciéndolas (Shapero, 1985).

\subsubsection{Factores endógenos}

Han sido estudiados también como motivaciones de tipo personal. García (1992), las denominó aspiraciones personales o motivaciones positivas de seguridad, de lucro, de servicio social, de independencia y el interés por las tareas directivas. Por su parte, para Autio y Kaurannen (1994), estas comprenden la necesidad de logro, la necesidad de independencia y el deseo de riqueza, las cuales pueden ser decisivas en la determinación de convertirse en emprendedor, siendo quizás, la más estudiada de estas, la necesidad o motivación de logro -abreviada como Nach, de Need for achievement-. Esta se relaciona también con el deseo de superación y progreso que lleva a los individuos a buscar siempre un estado superior en sus actividades (Varela \& Bedoya, 2006). Collins et al. (2004), concluyeron que se correlaciona significativamente con la elección de carrera y el rendimiento empresarial. En forma similar, Barba-Sánchez y Atienza-Sahuquillo (2011), han identificado que las personas con alta necesidad de logro quieren hacer bien las cosas y obtener resultados mejores que otros, incluyendo a los que tienen más así mismo, disfrutan tomando responsabilidades personales y recibiendo realimentación por sus acciones.

Quevedo, Izar y Romo (2010), realizaron un estudio para identificar los factores endógenos y exógenos de mujeres y hombres emprendedores en la constitución de su empresa. Los factores endógenos que identificaron son: audacia, pasión, creatividad, liderazgo, innovación, competitividad, intuición, empuje, persuasión, eficacia, capacidad de gestión y aventura. También, puede incluirse en estos factores la visión que el potencial emprendedor tiene de sí mismo; para Farmer, Yao \& Kung-Mcintyre (2011), el desarrollo de una visión de sí mismo como un emprendedor, representa una poderosa fuerza motivadora que puede ayudar a explicar por qué algunos individuos eligen y siguen participando en la actividad empresarial, y por qué otros no lo hacen. Es lo que en la teoría de la motivación se ha reconocido como autoeficacia percibida (Bandura, 1977).

\subsubsection{Factores exógenos}

La importancia del entorno en este proceso, como lo expone Douglass North (2006), está dada porque es el que impone las reglas de juego tanto formales como informales. Pero tan importante como estas reglas, es la percepción que sobre ellas tiene el potencial emprendedor, como afirman Mateo, Solves y Gras (2012): las variables de percepción se complementan con los aspectos propios del entorno como componentes críticos en la decisión emprendedora.

La teoría económica institucional, de Douglass North, plantea que los procesos 
económicos están insertados en un entorno social y cultural muy complejo. De acuerdo con esta teoría, las instituciones, como reglas del juego en una sociedad, son las «limitaciones ideadas por el hombre que dan forma a la interacción humana; por consiguiente, estructuran incentivos en el intercambio humano, sea político, social o económico» (North, 2006, p. 13). Así, estas reglas de juego -derechos de propiedad, legislación mercantil, trámites de constitución, ideas, creencias culturales, género, actitudes hacia el empresario, etc.-, influyen en la aparición y desarrollo de las nuevas empresas.

Tabla 1. Marco para el entorno empresarial.

\begin{tabular}{|c|c|}
\hline Políticas gubernamentales y los procedimientos. & Ayuda financiera. \\
\hline Restricciones en importaciones y exportaciones. & Capital de riesgo. \\
\hline Leyes de bancarrota. & Fuentes alternativas de financiación. \\
\hline Barreras de entrada. & Préstamos de bajo costo. \\
\hline $\begin{array}{l}\text { Requisitos y procedimientos para el registro y } \\
\text { concesión de licencias. }\end{array}$ & $\begin{array}{l}\text { Disposición de las entidades bancarias para financiar } \\
\text { pequeños emprendedores. }\end{array}$ \\
\hline $\begin{array}{l}\text { Número de instituciones a las que los empresarios } \\
\text { deben reportar. }\end{array}$ & $\begin{array}{l}\text { Programa de garantía de crédito para iniciar nuevas } \\
\text { empresas. }\end{array}$ \\
\hline $\begin{array}{l}\text { Normas y reglamentos que rigen la actividad } \\
\text { empresarial. }\end{array}$ & Competencia entre instituciones financieras. \\
\hline \multicolumn{2}{|l|}{ Leyes para proteger los derechos de propiedad. } \\
\hline Condiciones socioeconómicas & Asistencia no financiera \\
\hline Actitud del público hacia el emprendimiento. & Servicios de asesoramiento y apoyo. \\
\hline Presencia de empresarios con experiencia. & Redes empresariales. \\
\hline $\begin{array}{l}\text { Existencia de personas con características } \\
\text { emprendedoras. }\end{array}$ & Instalaciones de las incubadoras \\
\hline $\begin{array}{l}\text { Proporción de pequeñas empresas en el total de } \\
\text { empresas. }\end{array}$ & $\begin{array}{l}\text { Programas de compras gubernamentales para las } \\
\text { pequeñas empresas. }\end{array}$ \\
\hline Diversidad de actividades económicas. & $\begin{array}{l}\text { Apoyo gubernamental para investigación y } \\
\text { desarrollo. }\end{array}$ \\
\hline Nivel de crecimiento económico. & Incentivos y exenciones tributarias. \\
\hline Aptitudes empresariales y de negocios & Red de información local e internacional. \\
\hline Educación técnica y vocacional. & Medios de comunicación y transporte. \\
\hline Educación en temas de negocios. & \\
\hline Programas de formación empresarial. & \\
\hline Programas de entrenamiento técnico y vocacional. & \\
\hline Disponibilidad de información. & \\
\hline
\end{tabular}

Fuente. Elaboración propia de las autoras a partir de Gnyawali \& Fogel (1994, p. 46).
Basándose en esta teoría, Urbano y Díaz (2009) afirman que el entorno institucional en cada región o país será determinante en cuanto a las oportunidades disponibles - empresariales o no-; a la percepción que se tenga de ellas; al desarrollo de habilidades y capacidades para aprovecharlas además de que podrán formar parte de las motivaciones que desencadenan en la creación de una empresa. Gnyawali \& Fogel (1994), clasificaron las condiciones para crear empresa en cinco grupos: políticas gubernamentales y procedimientos, condiciones socioeconómicas, habilidades emprendedoras y de negocios, apoyo financiero y asistencia no financiera (Tabla 1). 


\section{Ecosistema emprendedor en Medellín}

Spilling (1996, p. 91), define el ecosistema emprendedor como «la complejidad y diversidad de actores, roles y factores ambientales que interactúan para determinar el desempeño empresarial de una región o localidad».

En Colombia, el SENA ${ }^{1}$ es una de las instituciones que lidera el programa de emprendimiento al cubrir todas las etapas

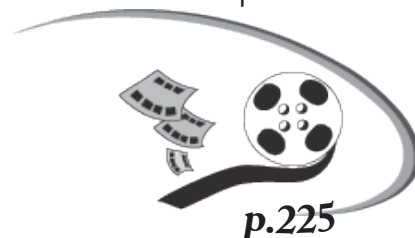
del proceso, desde el acompañamiento a la persona que manifiesta su deseo de ser emprendedor, pasando por la financiación y ayuda en la puesta en marcha, hasta la capacitación cuando las empresas están consolidadas.

En la ciudad de Medellín se ha ido construyendo, desde hace una década, un sistema de emprendimiento a través de alianzas público-privadas. Así, la Incubadora de Empresas de Base Tecnológica de Antioquia (IEBTA) fue creada en 1996 con el objetivo de generar cultura emprendedora y promover la creación y el fortalecimiento empresarial en Colombia -actualmente se denomina Creame-. Hacia el año 2000 se empezaron a desarrollar programas como Medellín Emprende, con el mismo propósito. Sin embargo, fue a partir de 2004, que el tema del emprendimiento se incluyó en el Plan de Desarrollo Municipal, el cual se basó en la estrategia denominada Cultura E (Yepes, 2013).

Más tarde, y como parte de esta estrategia, se creó el programa Ciudad E, que vinculó a las instituciones de educación superior (IES) y otras organizaciones, como las cajas de compensación familiar, agremiaciones, entre otros, para brindar soporte en el área de capacitación para el emprendimiento, así como el desarrollo de concursos que ofrecen como incentivo ayuda en la financiación de los proyectos ganadores.

Es de anotar que con los cambios de administración municipal también se han hecho cambios a la política de emprendimiento, es así que ya no existen Ciudad E y Cultura $\mathrm{E}$, como marcas, pero sí las instituciones y programas que las conformaron. A continuación se presenta un resumen de las principales instituciones y servicios relacionados con el fomento a la actividad emprendedora (Tabla 2).

1 El Servicio Nacional de Aprendizaje, SENA, es un establecimiento público del orden nacional, adscrito al Ministerio de Trabajo, que fue creado con el objetivo de capacitar a los jóvenes y adultos para el trabajo y prestar servicios enfocados en la generación de empleo. En este contexto, la entidad lidera el programa de emprendimiento y empresarismo más completo de Colombia. 
Tabla 2. Ecosistema emprendedor en Medellín.

\begin{tabular}{|c|c|c|c|}
\hline Tipo de servicio & $\begin{array}{l}\text { Emprendimiento } \\
\text { tradicional }\end{array}$ & Base tecnológica & Base cultural \\
\hline Asesoría & $\begin{array}{c}\text { Unidades de } \\
\text { emprendimiento IES } \\
{ }^{\star} \text { Cedezo } \\
\text { Interactuar } \\
\text { Famiempresas }\end{array}$ & $\begin{array}{l}\text { Unidades de emprendimiento } \\
\text { Tecnoparque } \\
\text { Parque E } \\
\text { Creame } \\
\text { Ruta N }\end{array}$ & $\begin{array}{c}\text { Cedezo } \\
\text { Empresas creativas y } \\
\text { culturales, Facultad de } \\
\text { Comunicaciones, U. } \\
\text { de A. }\end{array}$ \\
\hline Financiación & $\begin{array}{l}\text { Banco de las } \\
\text { oportunidades. } \\
\text { Interactuar } \\
\text { Famiempresas } \\
\text { *Fondo Emprender }\end{array}$ & $\begin{array}{l}\text { Ángeles inversionistas. } \\
\text { Fondo Emprender. }\end{array}$ & $\begin{array}{c}\text { Banco de las } \\
\text { oportunidades } \\
\text { Interactuar } \\
\text { Famiempresas } \\
\text { Fondo Emprender. }\end{array}$ \\
\hline Formalización & \multicolumn{3}{|c|}{ Cámara y Comercio, SENA } \\
\hline $\begin{array}{l}\text { Fortalecimiento } \\
\text { empresarial }\end{array}$ & \multicolumn{3}{|c|}{ Medellín, Ciudad Cluster, SENA } \\
\hline
\end{tabular}

Fuente. Elaboración propia de las autoras.

*Centros de Desarrollo Empresarial Zonal

El sistema de apoyo al emprendimiento en la ciudad está estratificado de acuerdo con el servicio y el tipo de emprendimiento.

\section{Metodología}

Con base en los planteamientos teóricos analizados, se diseñó un cuestionario para indagar sobre los factores que motivaron a emprendedores de la ciudad de Medellín a crear y administrar sus empresas, así como sus percepciones sobre las facilidades que se ofrece en dicha ciudad para el emprendimiento y sobre los obstáculos más grandes que

\section{Resultados}

\subsection{Perfil del emprendedor}

La muestra obtenida está conformada en un $56 \%$ por mujeres. Con respecto a la edad, la mayoría de entrevistados se encontraban entre los 25 y 35 años -56 \%-y un 28 \% eran deben superar. Dado que se trata de un estudio exploratorio, el cuestionario es semiestructurado y las preguntas por estos tres aspectos son abiertas. Este se aplicó a 18 emprendedores, la mayoría de ellos recibieron acompañamiento del programa de emprendimiento de la Universidad Nacional de Colombia, sede Medellín. Las encuestas se aplicaron personalmente. menores de 25 años. Por otra parte, el $61 \%$ contaba con formación profesional completa y un $17 \%$ en proceso de formación universitaria. Finalmente, el $82 \%$ de los participantes era soltero. Más adelante, se muestra un resumen de las principales características del perfil de los participantes (Tabla 3). 
Tabla 3. Perfil demográfico.

\begin{tabular}{|c|c|}
\hline Criterio & Participación \\
\hline \multicolumn{2}{|c|}{ Sexo } \\
\hline Femenino & $56 \%$ \\
\hline Masculino & $44 \%$ \\
\hline \multicolumn{2}{|c|}{ Edad } \\
\hline Menos de 25 años. & $28 \%$ \\
\hline Entre 25 y 35 años. & $56 \%$ \\
\hline Entre 35 y 45 años. & $17 \%$ \\
\hline \multicolumn{2}{|c|}{ Educación } \\
\hline Bachillerato & $6 \%$ \\
\hline Universitaria incompleta. & $17 \%$ \\
\hline Universitaria completa. & $61 \%$ \\
\hline Especialización & $5 \%$ \\
\hline Maestría & $11 \%$ \\
\hline Criterio & Participación \\
\hline \multicolumn{2}{|c|}{ Estado civil } \\
\hline Casado & $6 \%$ \\
\hline Soltero & $82 \%$ \\
\hline Otro (divorciado). & $6 \%$ \\
\hline NC & $6 \%$ \\
\hline
\end{tabular}

Fuente. Elaboración propia de las autoras.

\subsection{Perfil de las empresas}

Todas las empresas estudiadas son microempresas, esto es, tienen un máximo de diez trabajadores.

En cuanto a la actividad económica se encuentra una mayor heterogeneidad, pues participaron empresas de sectores tradicionales, como el de vestuario femenino, hasta empresas desarrolladoras de software. De acuerdo con esta situación, se realizó una clasificación como se muestra a continuación (Figura 2).
Figura 2. Distribución de empresas por actividad económica.

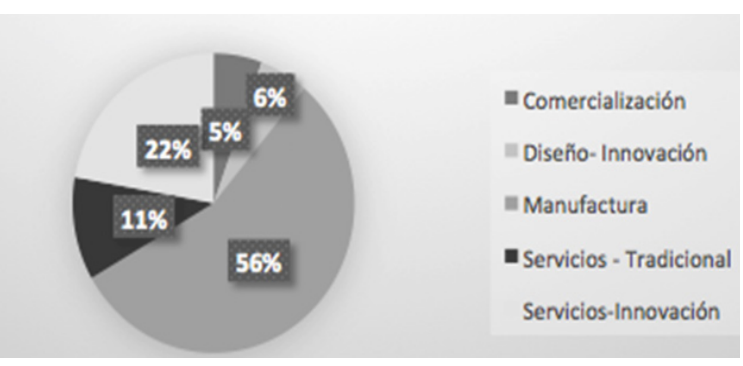

Fuente. Elaboración propia de las autoras.

\subsection{Motivaciones para emprender}

Tabla 4. Motivaciones para emprender.

\begin{tabular}{|l|c|}
\multicolumn{1}{c}{ Factor motivacional } & $\begin{array}{c}\% \\
\text { Independencián }\end{array}$ \\
\hline Dinero & $67 \%$ \\
\hline Tipo de negocio. & $61 \%$ \\
\hline $\begin{array}{l}\text { Contribución -empleo y crecimiento } \\
\text { del país-. }\end{array}$ & $33 \%$ \\
\hline Oportunidad & $22 \%$ \\
\hline Empleo & $22 \%$ \\
\hline Tiempo & $11 \%$ \\
\hline Familia & $11 \%$ \\
\hline Tener algo propio. & $11 \%$ \\
\hline Satisfacción personal. & $11 \%$ \\
\hline Autoeficacia & $11 \%$ \\
\hline Decisión de cambio. & $6 \%$ \\
\hline Crecimiento personal y profesional. & $6 \%$ \\
\hline Gusto por emprendimiento. & $6 \%$ \\
\hline Innovar & $6 \%$ \\
\hline
\end{tabular}

Fuente. Elaboración propia de las autoras.

La independencia fue mencionada como factor motivacional por el $67 \%$ de los encuestados, ubicándose como el más importante; en segundo lugar, aparece el factor relacionado con el dinero (Figura 3). 
Figura 3. Motivaciones para emprender.

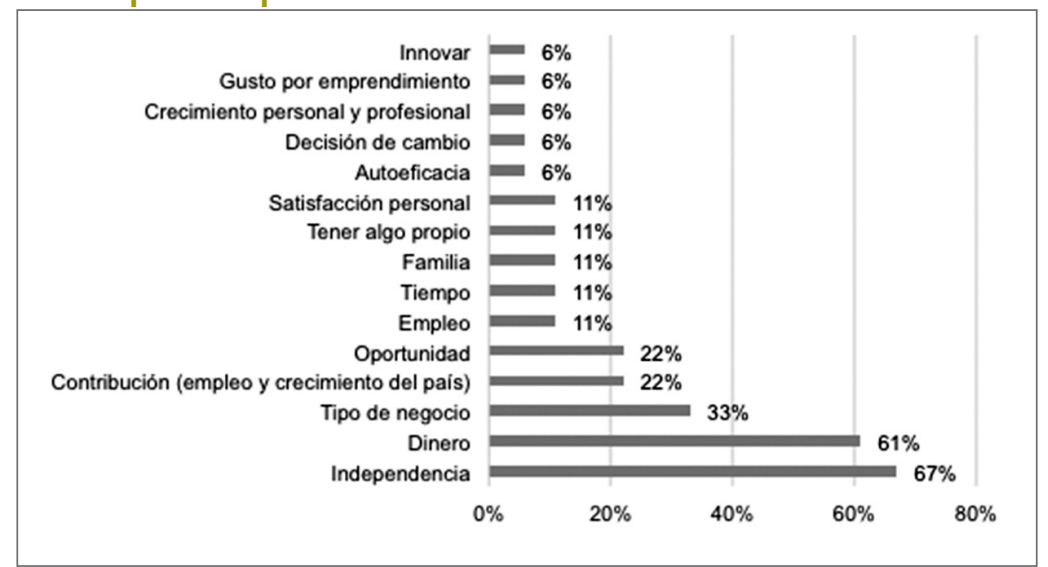

Fuente. Elaboración propia de las autoras.

Como puede observarse, el único factor exógeno que se menciona es la oportunidad.
En cuanto a los factores endógenos, la independencia y el dinero son los más representativos (Tabla 5).

Tabla 5. Facilidades que ofrece la ciudad de Medellín.

\begin{tabular}{|l|c|}
\multicolumn{1}{c}{ FACILIDAD } & $\begin{array}{c}\% \\
\text { MENCIÓN }\end{array}$ \\
\hline Acompañamiento o asesoría/capacitación. & $33 \%$ \\
\hline Concursos & $28 \%$ \\
\hline Entidades de apoyo/ecosistema. & $17 \%$ \\
\hline Beneficios legales. & $11 \%$ \\
\hline Publicidad & $6 \%$ \\
\hline Política de ciudad. & $6 \%$ \\
\hline Mentorías & $6 \%$ \\
\hline Apoyo económico. & $6 \%$ \\
\hline Ruedas de negocios. & $6 \%$ \\
\hline Información & $6 \%$ \\
\hline Ninguna & $6 \%$ \\
\hline
\end{tabular}

Fuente. Elaboración propia de las autoras. 
Figura 4. Facilidades que ofrece la ciudad de Medellín.

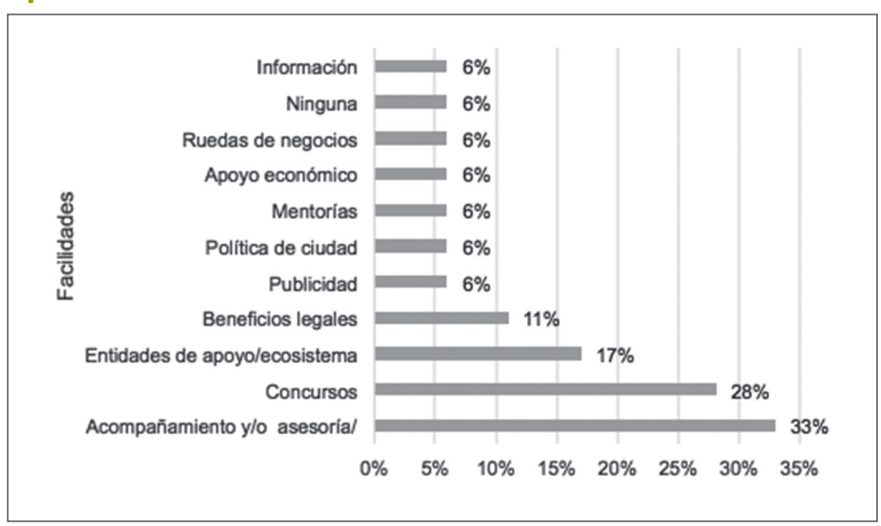

Fuente. Elaboración propia de las autoras.

Tabla 6. Principales obstáculos para emprender.

\begin{tabular}{|l|c|}
\multicolumn{1}{|c|}{ Dificultades } & $\%$ Mención \\
\hline Falta de financiación/capita.I & $50 \%$ \\
\hline Acceso a mercados. & $17 \%$ \\
\hline Idiosincrasia/mentalidad. & $11 \%$ \\
\hline Poca agremiación y trabajo en equipo. & $11 \%$ \\
\hline Tramitología & $11 \%$ \\
\hline Burocracia & $6 \%$ \\
\hline Canibalismo & $6 \%$ \\
\hline Contrabando & $6 \%$ \\
\hline Demasiado énfasis en plan de negocios. & $6 \%$ \\
\hline Falta acompañamiento. & $6 \%$ \\
\hline Miedo a asociarse. & $6 \%$ \\
\hline Monopolio & $6 \%$ \\
\hline Mucha competencia. & $6 \%$ \\
\hline Pereza & $6 \%$ \\
\hline Poca información. & $6 \%$ \\
\hline Poco reconocimiento de la experiencia. & $6 \%$ \\
\hline Proveedores no se ajustan a pedidos. & $6 \%$ \\
\hline Uno mismo se crea obstáculos. & $6 \%$ \\
\hline
\end{tabular}

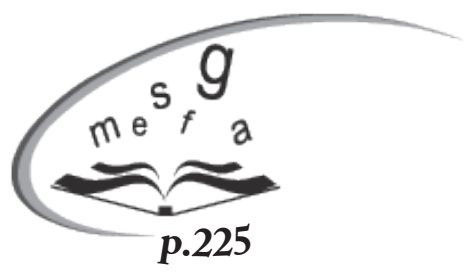

Fuente. Elaboración propia de las autoras. 
Figura 5. Principales obstáculos para emprender.

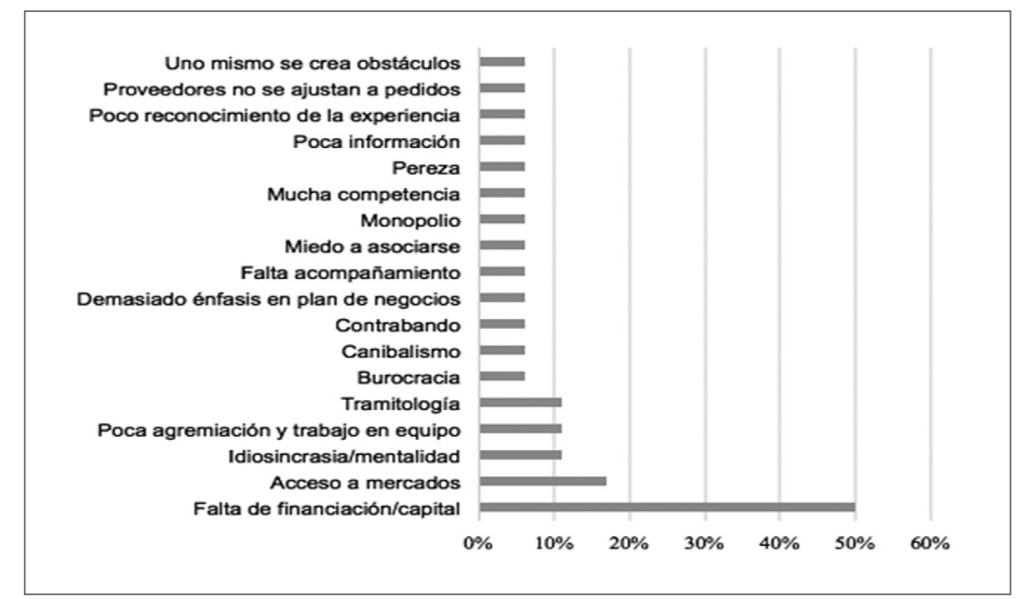

Fuente. Elaboración propia de las autoras.

\section{Discusión}

L

os resultados dejan en evidencia que la principal motivación para emprender es la independencia, lo cual es coherente con los planteamientos de Shapero (1985), quien la describe como la motivación dominante para convertirse en empresario. El término tiene una acepción muy amplia en cuanto al tema del emprendimiento. Manolova et al. (2008), lo denominan autonomía y lo describen como un deseo individual de libertad, control y flexibilidad en el uso del tiempo. Incluye aspectos como el anhelo de un individuo de planear su trabajo y tomar sus propias decisiones (Carter et al., 2003; Raposo et al., 2008). Shane et al. (2003), afirman que la independencia implica tener la responsabilidad de usar su propio juicio en lugar de seguir ciegamente las afirmaciones de los demás, así mismo, asumir la responsabilidad de la propia vida en lugar de vivir de los esfuerzos de otros.

En este estudio, el aspecto económico fue el segundo motivador, con una mención del $61 \%$. Este resultado coincide con lo reportado en la literatura, en donde se ha encontrado que el deseo de riqueza es uno de los objetivos principales de los emprendedores (Raposo et al., 2008). Edelman, Brush, Manolova y Greene (2010), le denominan éxito financiero, que implica las intenciones de los individuos de ganar más dinero y lograr la seguridad financiera. Carter et al. (2003), tienen en cuenta aspectos como ganar seguridad para sí mismo, el cónyuge e hijos y contribuir al bienestar de los familiares.

En general, en la decisión de convertirse en emprendedores, en este estudio predominó la motivación intrínseca en el sentido de que esta se origina dentro del sujeto, a través de la autopercepción como persona competente, eficaz y con determinación para actuar, es decir, es el individuo el agente causal de su propia actividad comportamental, tal como lo expuso Barberá-Heredia (2002). De los factores motivacionales externos, se encontró solamente que la oportunidad incidía en el $22 \%$ de los casos. 
Sin embargo, al indagar por la percepción de las facilidades que ofrece el entorno, y teniendo en cuenta que se trató de una pregunta abierta, se nota que los emprendedores reconocen que se hace un esfuerzo por impulsar esta actividad. El aspecto que ellos consideran más influyente es el relacionado con el acompañamiento que brindan las diferentes instituciones comprometidas con esta labor, ya sea a través de asesoría, contactos o capacitación.

También, consideran de gran importancia los diferentes concursos que se hacen en la ciudad, como el Capital Semilla, Jóvenes emprendedores, Fondo Emprender, Mujer talento, etc. Algunos manifestaron que no habían podido acceder a ninguno de estos fondos, pero reconocen que son valiosos.

Así mismo, se mencionaron aspectos como las mentorías y las ruedas de negocios, que son programas diseñados para empresas en funcionamiento que necesitan fortalecerse. En este sentido, aplican las conclusiones de Sastre (2013), quien explica que los programas de apoyo tanto de capacitación como de recursos, parecen ser más necesarios cuando el emprendimiento se encuentra en su fase de consolidación.

Los aspectos legales tuvieron mención del $11 \%$, tanto en facilidades como en barreras. Este aspecto es el que tiene que ver con las reglas de juego institucionales (North, 2006). Quienes los mencionaron como facilidad se referían a exenciones tributarias, y quienes los consideraron como obstáculo, hacían referencia a que se les exige el mismo volumen de trámites e informes para las distintas entidades estatales, que a las grandes empresas. Esto coincide con la afirmación de Shapero en cuanto a que el efecto principal del entorno político-legislativo-administrativo es negativo (Shapero, 1985).

Es justamente esta situación la que hace que los factores personales, especialmente los más relacionados con motivación de logro, sean los más importantes, pues como lo plantean Stewart \& Roth (2007), son los individuos más decididos y persistentes los que logran crear, consolidar y mantener sus empresas. De otro lado, no todos los factores del entorno son negativos o de poca importancia; como ya se mencionó, los programas que buscan la consolidación y permanencia de las empresas son de gran ayuda cuando el negocio está operando; además, en Medellín y su Área Metropolitana tienen una buena cobertura. En este sentido los resultados son acordes con los de Sastre (2013).

El aspecto que tiene la percepción más negativa es el de la financiación. Fue mencionada por el $50 \%$ de los encuestados, quienes manifestaron que han tenido pocas o nulas posibilidades de acceso al mercado financiero en la etapa inicial y aún en la actualidad cuando sus empresas ya se encuentran en funcionamiento. $Y$ es que las pocas posibilidades de crédito se les ofrecen en condiciones muy poco competitivas, y es precisamente la falta de capital el principal problema que aqueja a los emprendedores. En Colombia existe una línea de crédito de fomento para el emprendimiento conocida como microcrédito, pero las tasas que manejan están en entre el $35 \%$ y el $45 \%$, valores desproporcionados que no contribuyen a la liquidez de las pequeñas empresas. Las entidades financieras que cobran las tasas más altas lo justifican diciendo que prestan un servicio muy ágil, lo que hace que aquellos empresarios con necesidades financieras más apremiantes tengan que aceptar esas condiciones. 
El acceso a mercados fue otro de los obstáculos mencionados por los encuestados. Teniendo en cuenta que la mayoría de las actividades económicas a las que se dedican son tradicionales -confecciones, accesorios femeninos-, este aspecto está muy relacionado con la competencia que existe en el medio para este tipo de productos. Incluso se ha incrementado fuertemente en los últimos años tanto por vías legales, como los tratados de libre comercio, como por las ilegales, el contrabando.

\section{Conclusiones}

Cegún se desprende de los resultados del $\checkmark$ presente estudio podría decirse que las campañas de fomento al emprendimiento están dando resultados. Esto puede notarse en varios aspectos:

- El perfil de los emprendedores muestra que la mujer va ganando participación en la actividad.

- El nivel de formación de quienes eligen el emprendimiento como carrera, también ha ido progresando, y cada vez más profesionales, incluso con estudios de posgrado, se dedican a esta actividad.

- Los emprendedores reconocen que se hace un esfuerzo desde diferentes entidades por ofrecer actividades de apoyo tendientes a facilitar la sostenibilidad y el fortalecimiento de las empresas.

En cuanto a las motivaciones para emprender, se halló que las intrínsecas son las que tienen una mayor influencia destacándose entre ellas el deseo de independencia. El aspecto económico también fue un factor determinante. Al integrar este resultado con el perfil descrito, especialmente en lo relacionado con la formación, se nota que no se trata de un emprendimiento por necesidad, sino que es la actividad que ellos escogieron como carrera y es apenas natural que esperen vivir de ella.

Más allá de las actividades de capacitación y acompañamiento, se requiere, por parte del gobierno y las entidades financieras, una verdadera voluntad de apoyo al emprendedor, pues líneas de crédito como las existentes, lejos de ayudar, lo que hacen es volver más crítica la situación económica del emprendedor, quien además de sus obligaciones normales, tiene que asumir las difíciles condiciones de un crédito que la única ventaja que le ofrece es recibir el dinero en corto tiempo.

También es importante considerar el tamaño de las empresas al momento de crear leyes, especialmente con relación al volumen y a la complejidad de los informes que se deben presentar a los diferentes organismos de control y vigilancia. Esto no va en contraposición con la legalidad y formalización que deben tener dichas empresas, sino que debe adecuarse a las facilidades que ellas tengan para contratar a las personas y los sistemas necesarios para responder en forma oportuna con los requerimientos. 


\section{Referencias bibliográficas}

Autio, E., \& Kauranen, I. (1994). Technologistentrepreneurs versus non-entrepreneurial technologists: analysis of motivational triggering factors. Entrepreneurship \& Regional Development, 6 (4), 315-328.

Bandura, A. (1977). Self-efficacy:Toward a Unifying Theory of Behavioral Change. Psychological Review. 84(2), pp. 191-215.

Barba-Sánchez, V., y Atienza-Sahuquillo, C. (2011). Reasons to create a new venture: A determinant of entrepreneurial profiles. African Journal of Business Management, 5(28), 11497-11504.

Barberá-Heredia, E. (2002). Modelos explicativos en psicología de la motivación. Revista Electrónica de Motivación y Emoción 5(10), pp. 6.

Capelleras, J. L., Contín-Pilart, I., MartínSánchez, V., \& Larraza-Kintana, M. (2013). The influence of individual perceptions and the urban/rural environment on nascent entrepreneurship. Investigaciones regionales (26), pp. 97-113.

Carsrud, A., \& Brännback, M. (2011). Entrepreneurial Motivations: What Do We Still Need to Know. Journal of Small Business Management, 49(1), 9-26.

Carter, N. M., Gartner, W. B., Shaver, K. G., \& Gatewood, E. J. (2003). The career reasons of nascent entrepreneurs. Journal of Business Venturing 18, pp. 13-39.

Collins, C. J., Hanges, P. J., \& Locke, E. A. (2004). The Relationship of Achievement Motivation to entrepreneurial Behavior: A Meta-Analysis. Human Performance, 17(1), pp. 95-117.
Edelman, L., Brush, C., Manolova, T., \& Greene, P. (2010). Start-up Motivations and Growth Intentions of Minority Nascent Entrepreneurs. Journal of Small Business Management 48(2), pp.174-196.

Farmer, S. M., Yao, X., \& Kung-Mcintyre, K. (2011). The Behavioral Impact of Entrepreneur Identity Aspiration and Prior Entrepreneurial Experience.

Entrepreneurship Theory And Practice, pp. 245-273.

García, E. E. (1992). Incidencia de las motivaciones personales en el proceso de creación de la nueva pyme industrial. Revista Europea de Dirección y Economía de la Empresa, 1(1), pp. 67-78.

Gnyawali, D., \& Fogel, D. (1994). Environments for Entrepreneurship Development: Key Dimensions and Research Implications. Entrepreneurship, Theory and Practice, 18(4), pp. 43-62.

Kantis, H., Angelelli, P., \& Gatto, F. (2001). Nuevos emprendimientos y emprendedores: de qué depende su creación y supervivencia. Explorando el caso argentino, UNGSLITTEC, DT, 2. Buenos Aires: Littec.

Manolova, T., Brush, C., \& Edelman, L. (2008). What do women entrepreneurs want? Strategic Change, 17 (3-4), pp. 69-82.

Marulanda, F. Á. (2015). Una contribución a la comprensión de las motivaciones del emprendedor y su conexión con el emprendimiento MIPES de valle de Aburrá. Medellín: Universidad Nacional de Colombia.

Mateo, J. M., Solves, I. M., \& Gras, J. M. (2012). Influencias de las percepciones personales 
sobre la actividad emprendedora naciente: una perspectiva de género a través de diferentes entornos económicos. Economía industrial, 383, pp. 53-64.

McClelland, D. (1961). The Achieving Society. New York: A Free Press Paperback.

McClelland, D. (1965). Thinking aheadAchievement Motivation Can Be Developed. Harvard Business Review, pp. 7-24.

MEN, C. M. (2006). Ley 1014 de 2006. Recuperado de: <http://www.mineducacion.gov.co/1621/article-94653.html>

Méndez, C., \& Rondón, M. A. (2012). Introducción al análisis factorial exploratorio. Revista Colombiana de Psiquiatría. 41 (1), pp. 197-207.

North, D. C. (2006). Instituciones, cambio institucional y desempeño económico. México: Fondo de Cultura Económica.

Orrego, C. (2008). La dimensión humana del emprendimiento. Revista Ciencias Estratégicas. 16 (20), pp. 225-235.

Palmero, F. (2005). Motivación: conducta y proceso. Revista Electrónica de Motivación y Emoción. 8 (20-21), pp. 1-29.

Paturel, R. (1997). Pratique du management straégique. Grenoble: Presses Universitaires de Grenoble.

Quevedo, L. M., Izar, J. M., \& Romo, L. (2010). Factores endógenos y exógenos de mujeres y hombres emprendedores de España, Estados Unidos y México. Investigación y Ciencia. 18 (46), pp. 57-63.

Sastre, R. (2013). La motivación emprendedora y los factores que contribuyen con el éxito del emprendimiento. Ciencias Administrativas, 1, pp. 1-10.
Shane, S., Locke, E. A., \& Collins, C. J. (2003). Entrepreneurial Motivation. Human Resource Management Review. 13, pp. 257-279.

Shapero, A. T. (1985). The entrepreneurial event. Ohio: College of Administrative Science, Ohio State University.

Spilling, O. R. (1996). The entrepreneurial system: On entrepreneurship in the context of a mega-event. Journal of Business research, 36 (1), pp. 91-103.

Stewart, W., \& Roth, P. (2007). A Meta-Analysis of Achievement Motivation Differences between Entrepreneurs and Managers. Journal of Small Business Management 2007, 45 (4), pp. 401-421.

Universidad de Medellín, Fundación ECSIM, Universidad Pontificia Bolivariana; Universidad Javeriana de Cali, Secretaría de Productividad y Competitividad; Departamento de Antioquia. Global Entrepreneurship Monitor Antioquia 2012. Medellín: Gobernación de Antioquia.

Urbano, D., y Díaz, J. C. (2009). Creación de empresas e instituciones: un modelo teórico. En M. F. R. Hernández, Creación de empresas. Aproximación al estado del arte. Lisboa: Juruá pp. 95-109.

Varela, R., y Bedoya, O. (2006). Modelo conceptual de desarrollo empresarial basado en competencias. Estudios gerenciales, 22(100), pp. 21-47.

Yepes, M. (2013). Ciudad E ecosistema de emprendiemiento de Medellín. Medellín: Universidad EAFIT 